\title{
SUCCESSFUL ERRORS AND OTHER ODD WAYS TO NEW DISCOVERIES*
}

\author{
by
}

\section{F. LEMBECK}

MediCAL RESEARCH tends more and more to become a technical process. This includes not only the technical equipment of our laboratories, but also the co-operation of specialists coming from different fields of medicine and natural science. Co-operation affords organisation and administration. When such work has to be done by a scientist, he has to leave the laboratory and occupy chairs on committees. This may severely change his outlook on scientific life and productivity, a fact which Anne Roe recently brought to our notice in an interesting comment in Science.

Governments and other organizations develop research programmes and think in terms of new buildings, of financial support and of the publication of results. These basic prerequisites are certainly essential to research programmes. Scientists must become accustomed to co-operate in such programmes. Large programmes are often very successful; for example, the search for antimalarial drugs or the screening of new antibiotics and their clinical trials. Under such conditions the specialists confronted with a specific problem become a team, often a very effective one, and not a 'lost legion' as the occasionally large number of scientists involved in a single programme in physics or technology have been called.

This general picture of large-scale research may, however, also change our individual approach to research problems. Some very original characteristics of the men who formerly undertook 'one-man expeditions' may be lost, but, at each stage, and each time research is attempted, completely unexpected results may occasionally be obtained. During a planned research programme which has to reach a certain goal such side-effects of research may easily be neglected. We in university laboratories are relatively free from an excessive amount of programming, but a drawing from an industrial firm can show you the seven years of intrauterine development of a new drug. It includes the function of the pharmacologist, not unlike that of a transfer RNA, who selects chemical information and carries it to the therapeutic application. Previous 'findings' have been transformed into 'constructions' like an aircraft or a bridge, as we see when we look at the final result.

Ideas which by way of experiment lead to new discoveries are stimulated in different ways:

1) Nature itself offers many examples of models for later technical application: An aircraft looks and glides like a bird. Even the wing mechanism of a helicopter is mechanically equivalent to the wing and feather movements of a bird when it is fluttering. 2) Other discoveries are purely the result of the human brain: Circular movements occur in Nature, but the wheel has been found only above a certain level of civilisation, and, for instance, was not familiar to the otherwise intelligent and

- A lecture given at the Middlesex Hospital Medical School on 12 May 1966. 


\section{F. Lembeck}

skilful Maya. 3) Many physical or chemical processes are used by living organisms: their mechanism could not be readily understood by the human mind before their physical or chemical basis had been established. In some instances these processes had been put to technical use before it was recognized that they were also in natural use. Ultrasonic echo waves were already being used in commercial fisheries when it was discovered that bats used them for orientation. Even the modern fluid-propelled rockets have their involuntary predecessors in Nature. The insects of the species Brachynus Bombardier beetle produce a 25 per cent solution of hydrogen peroxide and hydroquinone in a gland and collect this mixture in a bladder. A valve opened by a muscle allows the mixture to enter a chitin shielded sort of combustion chamber. Here an explosive catalytic reaction produces quinone and liberates oxygen which expels the fluid through an outlet valve at very high speed. 4) Occasionally, a discovery is the result of an incidental mistake or the neglect of rules. Blotting paper, now used in the advanced form of filter paper for chromatography, was first produced because someone forgot to add glue to the paper pulp, whereupon he was dismissed from the paper mill. The discovery of Ringer solution occurred because Ringer's technician found the London tap water as good as distilled water for preparing a saline solution. During his technician's absence, Ringer made saline himself and involuntarily observed that distilled water was not as good as the water which the technician had used.

On the way from a hypothesis to a new discovery three possibilities occur:

1) We know examples of a profound idea resulting in clear experimental evidence. This may be regarded as the straight line of success. 2) We also know that a correct hypothesis is not necessarily successfully proven by experimental evidence. 3) Finally, quite unexpected results may appear without any connection to the original hypothesis.

As an example of the first possibility consider Paul Ehrlich's drawings of 'Haptophore' and 'Toxophore Gruppen'; these 'schöne Figuren' are even now, when we study molecular aspects of receptor-drug interactions, of impressive value.

To illustrate the second possibility we can cite Zülzer who, earlier than Best and Banting, had probably discovered the right method of extracting insulin from the pancreas, but he injected too much of his extract into the rabbits and they died from convulsions.

Feyrter, very early, had the idea that carcinoids liberate an active endocrine substance; but from post-mortem findings he could not reconstruct the clinical picture of the carcinoid syndrome. He recommended the injection of a carcinoid extract into an animal. This led to a blood pressure response in the cat which suggested the presence of an adrenaline-like compound. When he carried out a post-mortem examination of a carcinoid case he sent his assistants to the relatives of the case to enquire about his life and possible symptoms. From these enquiries he deduced some connection between carcinoids and alcoholism. (I personally think that many of these cases had a red face or a flush which was most likely to be explained by the relatives as a result of a high alcohol consumption.)

Another example of this kind is seen in the work of Laland and Klem who, as early as 1939, produced a very refined preparation of the anti-pernicious anaemia 


\section{Successful Errors and Other Odd Ways to New Discoveries}

factor. At that time further work was interrupted. A sample of their preparation was placed on a slide under a cover glass. Nineteen years later, others put this slide under a microscope and observed the typical red crystals of cyanocobalamin.

As for the third possibility, in recent years there have been so many straight lines from the hypothesis to the expected result that this is now the most usual procedure. I want, however, to present a strange collection of examples in which discoveries have been made on the basis of wrong assumptions, neglected facts, incidental events-in short, where the success of the research depended on some sort of error in the planning, the work, or the conclusions.

Before 1900 Liebermann introduced phenolphthalein for masking the colour of adulterated wines made from the husks of grapes. This colouring prevented the wine from being mixed and sold with wines of good quality. $10 \mathrm{mg}$. of phenolphthalein per litre of wine is sufficient to give a deep red colour upon addition of sodium hydroxide. Vanossy in Budapest thereafter performed a chronic toxicity test of phenolphthalein by using three rabbits and giving 30,100 and $200 \mathrm{mg}$. daily for two months. When he was thoroughly confident that the substance was harmless to animals, he gave it to 'healthy men, first of all himself' in the dose of $1.5 \mathrm{gm}$. This led to the discovery of its laxative action. But he stated 'in the interest of alcoholics', that the addition of $10 \mathrm{mg}$./litre of wine can be regarded as safe, 'even when 5-10 litres of wine are consumed daily'. Phenolphthalein has a laxative effect only in man and monkeys and it was not until 1941-48 that Loewe investigated a number of structural analogues in Rhesus monkeys. It was probably an error to look for protection of the alcoholics from wines of bad quality by adding phenolphthalein, but the use of phenolphthalein turned out to be of considerable help in treating constipation.

Disulfiram (Antabuse) has been used for years in the rubber industry as an antioxidant. It was also used as a skin disinfectant and as a fungicide. Hald, Jacobson and Carsen (1948) performed animal experiments and toxicity studies to introduce disulfiram as an anthelmintic. Stimulated by the low toxicity in animals they performed an experiment on themselves and enjoyed the apparent absence of toxic effects. Then, it has been told, they went to a cocktail party-thereafter feeling quite intoxicated. They knew the similar hypersensitivity to alcohol after contact with cyanamide or ingestion of the fungus Coprinus atramentarius, but the smell of acetaldehyde led them subsequently to find the mode of action of disulfiram. I think this is a remarkable success for all those who succumb to the error of regarding large doses of alcohol as an essential foodstuff.

According to Straub's version, Jarisch, the late pharmacologist of Innsbruck, felt that he belonged to the lower species of pharmacologists, those who use drugs only to explore physiological functions. One day he wished to advance to the higher species of pharmacologists who use experiments to discover the therapeutic action of a drug. He found himself impressed by some old reports about an antihypertensive effect of mistletoe (Viscum album) and chose to investigate it. He prepared extracts of it and observed a lowering of the blood pressure, which was at that time accounted for by some others by a presumed occurrence of acetylcholine in mistletoe. He revealed the reflex mechanism of mistletoe extracts on the heart. During the grinding of the mistletoe powder in preparing the extract, his co-worker, Henze, frequently 


\section{F. Lembeck}

had to sneeze. Since sneezing is also a reflex, Jarisch looked into some older literature concerning Veratrum (which is called 'sneeze root' in German) and found the almost forgotten cardiac reflex observed by Bezold in 1867 after injection of Veratrum extracts. These investigations led to the detailed description of the well-known JarischBezold reflex. In other words, Jarisch failed to move up to the so-called higher species of pharmacologists, but successfully used mistletoe to discover an important reflex mechanism.

Concerning the detection of the Dale-Schultz reaction, the best I can do, is to refer to Sir Henry Dale's own words:

I had become familiar, in other connections, with the use of the isolated uterus of the virgin guinea-pig as a sensitive sample of unstriated muscle. I was using it for some not immediately fruitful comparisons of the stimulating activity of freshly separated blood sera, of which horse serum was the one most easily available to me, with that of histamine; and thus I encountered a uterus, from a particular guinea-pig, which contracted rapidly and to a maximal extent in response to a first application of horse serum, in a normally quite inactive dilution. I was working in a laboratory in which many guinea-pigs were, of course, being used for assays of antitoxin, involving very small injections of horse serum; and I naturally remembered Theobald Smith's discovery, a few years earlier, of anaphylaxis to horse serum in guinea-pigs which had recovered from such tests. My then young colleague, A. T. Glenny, was in charge of this serum testing, and for that reason I had made him responsible for the supply of guinea-pigs to all users. Enquiry immediately confirmed my suspicion; Glenny had supposed that a guinea-pig surviving from an antitoxin test would be 'good enough' for my 'pharmacological experiments!'

This could not have happened in a laboratory where only fresh animals were used in every experiment.

After his famous Africa expedition in 1865, Livingstone reported on an arrow poison made from Strophanthus seeds. Kirk, who was one of his companions, first noticed its cardiac action. For some unknown reason Kirk's toothbrush became contaminated with this poison. He noticed the bitter taste and assumed a contamination with quinine. But then he observed cardiac effects and bradycardia. The subsequent investigation by Sharpey and his co-workers revealed its digitalis-like action. Sir Thomas Fraser performed comprehensive pharmacological and clinical studies. But it was not before 1906, that a practitioner in Baden, Germany, Albert Fränkel, introduced-against the advice of Schmiedeberg -intravenous Strophanthin therapy in the medical clinic of Heidelberg. We as human beings living in laboratories would probably say that it was rather careless that a toothbrush became contaminated with an arrow poison. But what a lucky accident!

The antifibrillatory action of quinine was first reported by Wenckebach in 1914 but was described by a patient:

... In 1912, a patient presented himself in my office wishing to get rid of his attacks of auricular fibrillation ... On my telling him that I could promise nothing, he told me that he knew himself how to get rid of his attacks but I did not believe him. He promised to come back next morning with a regular pulse, and he did ... I was greatly struck by this fact and afterwards tried this treatment on many cases of auricular fibrillation. My success was disappointing, in that quinine abolished auricular fibrillation in only a few cases, and in these cases only when the onset was quite recent, never when it was of several years duration.

Critical people may regard the patient as the real discoverer. We may, however, submit that the reputation of Wenckebach as a clinician encouraged the patient to tell 


\section{Successful Errors and Other Odd Ways to New Discoveries}

him about his secret remedy. This illustrates how successful a physician can be when he carefully listens to unexplained and often confusing reports of a patient.

Domagk, Klarer and Mietsch observed in 1932 that mice with streptococcal infection could be protected by the azo-dye prontosil. This compound had been developed in a series of azo-dyes. As early as 1903 Hoerlein synthesized the first colour-fast dyestuff with a sulphonamide group. The combination of azo-dyes with proteins led to the search for further substances which react with bacterial proteins. The bactericides chrysoidine, pyridium and scarlet red were found and the last two substances applied in man. The liberation of an active sulphonamide from chrysoidine was postulated, the high bactericidal action was noted, but the research was continued on the line of azo-dyes. Work on the azo-compounds achieved success with the important discovery of protonsil rubrum in 1932 . It subsequently turned out to be an error to believe that the 'azo' group was responsible for the effect, as in 1936 Fourneau discovered that sulphanilamide (protonsil album) was the active group in protonsil rubrum. He thereby gave a new and promising direction for further experimentation. A similar comment could be made on the discovery of anti-emetics among antihistamines, Monoamine oxidase inhibitors among tuberculostatics, saliuretics and antidiabetic drugs among sulphonamides, and the taeniacidal effect of atebrin.

The members of the philosophical society of Socrates were forbidden to eat Vicia fava beans. The reason for this rule remained for a long time unexplained. It is known, however, that some persons in Mediterranean countries suffered from haemoglobinuria when they came into contact with these plants. This was termed favism and regarded as a peculiar form of hypersensitivity. Recent findings showed that this sensitivity occurs only in those people whose erythrocytes lack the enzyme glucose-6phosphate dehydrogenase. These people also very often react to pamaquine, primaquine and other drugs with haemolysis. On the other hand, they are much more resistant to infection with Plasmodium falciparum than others. In malarial districts this is a reason for the expectation of longer life and better reproduction. The enzyme deficiency occurs therefore more frequently in malarial areas. We see an 'inborn error of metabolism' constituting an advantage when life is influenced by malaria infections. Even nature can be successful by producing a genetic error. How many errors may have been made in the development of homo sapiens?

The main pharmacological effects of 5-hydroxytryptamine were described in 1940 by Erspamer, at a time when the chemical structure was unknown and the compound was called 'Enteramine'. In 1947, Page and his co-workers began to isolate a vasoconstrictor and hypertensive agent from beef serum, arguing a possible significance of this compound in hypertension. By using the rabbit ear as a test preparation for vasoconstriction, they were successful in isolating serotonin, which is the same substance as Erspamer's enteramine. This discovery very soon led us to forget that serotonin is by no means a pathogenetic factor in hypertension which was anticipated at the very beginning of Page's research.

In 1962 Kirberger, working in Frankfurt at a hospital for the mentally ill, investigated the 5-hydroxyindolacetic acid (5-HIAA) excretion in a large number of psychotic patients. One of these patients excreted high amounts of 5-HIAA. Repeated estimations showed a 3 or 4 days' cycle of very high and low values. This cycle was 


\section{F. Lembeck}

found to bear no correlation to any symptoms but rather to the days when visits were allowed. At each visit the patient's mother brought him a parcel of nuts which he liked very much. This small gift prompted the discovery that nuts contain high amounts of 5-hydroxytryptamine. The planned experiments did not bring the result expected, but a useful observation for all those who perform 5-HIAA estimations in urine.

Examples of different kinds are widely scattered: Hahnemann is known as the founder of a system of medical treatment called homeopathy. For more than a century it seemed to be a riddle that medicines in very high dilutions, free from any detectable pharmacodynamic effect and-in the so called 'high potencies' probably free of any active molecules-could relieve symptoms and pain from a patient. Certainly, many drugs of the old allopaths were by no means better. Even today clouded evidence of homeopathic medicines is sought by some. We know that belief in these effects is an error, but hardly a better and more prolonged successful trial of placebo effects could be found.

Other examples can be found in various clinical disciplines: Barraquer, a Spanish ophthalmologist, had a patient with a severe haemorrhage in the vitreous body of the eye. Treatment was not possible and he thought as a last resort that injection of chymotrypsin into the eye might dissolve the blood clot. The next day, he observed no improvement but noted that the lens lay disconnected from its zonula fibres. Therefore he dropped chymotrypsin into opened eyes, first in rabbits, then in man, when he performed cataract operations, and found it thereafter to be extremely easy to extract the lens. In this way the enzymatic zonulolysis was found, now mainly performed by the use of trypsin.

Vogl (1950) described the discovery of the mercurial diuretics when he was a young doctor at the Wenckebach Clinic in Vienna in 1919. A patient with severe symptoms of congenital syphilis should, 'ut aliquid fieri', be injected daily with salicylate of mercury. Vogl had to write the prescription. But his prescription, a 10 per cent solution of mercurial salicylate, could not be provided by the hospital pharmacy because of its insolubility in water. While he was writing a new prescription a colleague offered him a new mercurial antisyphilitic which he had received as a sample for medical trial by mail that morning, under the proprietary name Novasurol. In this immediate post-war period, the training of nurses was being carried out in this clinic with scrupulous attention to detail, so scrupulous in fact that even urine was collected daily from all patients, measured, and recorded on the patient's chart. In this way the observation of the diuretic effect of Novasurol was made. Novasurol was first assumed to have a curative effect on the syphilitic kidney. Thereafter the diuretic action was also found in patients with non-syphilitic congestive heart failure. It was an error to believe in an antisyphilitic effect of Novasurol, but an unnecessary recording of urine output and a lack of proper writing of prescriptions led to the discovery of its diuretic action.

For many years Amidopyrine has been a frequently used antipyretic drug in many countries. It has been derived from Antipyrine. Antipyrine was synthesized by Knorr in 1884. He first tried to synthesize a quinine-like compound. But the concept of the

quinine formula was wrong at that time. The assumed formula of the new compound 


\section{Successful Errors and Other Odd Ways to New Discoveries}

that he obtained was wrong too; Knorr thought it would be dimethylquinoline. The clinician Filehne found that it had an excellent antipyretic action in man and the compound got the name antipyrin. Shortly thereafter, Knorr showed that his substance had a pyrazole ring and he published the correct structure.

Jancsó and Jancsó obtained in 1934 results which suggested that the trypanocidal action of suramin (Germanin) depends on the inhibition of carbohydrate metabolism in the trypanosomes by this chemotherapeutic agent. They assumed that a low glucose level in blood could inhibit the growth of trypanosomes. To investigate this, they injected the hypoglycaemic agent Synthalin. The chemotherapeutic result was excellent and they wrote in the summary of the paper: 'The first time a chemotherapeutic agent could be detected, not by incidence, but based on theoretical assumptions'. Yorke, however, found shortly thereafter a very strong trypanocidal action of Synthalin in vitro. Synthalin, as is well known, has no effect on the glucose content of blood in vitro! Other forms of hypoglycaemia were found to be without effect on trypanosomes. We see in this example that the firmly stated theoretical conclusion was an error and the pure accident that the researchers used Synthalin and not Insulin for lowering the blood glucose level opened the successful way to the investigation of further trypanocidal substances.

In these days, the investigation of new drugs is carried out most carefully by the large pharmaceutical firms. The industry performs long and careful tests to exclude any kind of damage to patients and to diminish the danger of having to withdraw an expensively developed drug from the market. But even here, unexpected events can occur.

Ethyl Biscoumacetate (Tromexan) was found during a screening of anticoagulants in rabbits with the aim of finding short-acting compounds. The action of Biscoumacetate in man was also found to be relatively short. However, there is no direct relation since metabolism in man is quite different from that in a rabbit. In contrast to human beings and rabbits which have a faster turnover, Biscoumacetate is metabolised at a rate of only 3 per cent per hour in the dog. What would have happened if the screening had been done in dogs? We can deduce from such examples that some sort of error may, on the other hand, easily prevent new discoveries.

Unexpected findings are as possible today as a hundred years ago. Recently it has been reported that thalidomide, given as a sedative to patients with severe lepra reactions, produces a rapid improvement in all the cases treated. A short time ago, Dr. Slama of Prague, brought the eggs of an insect, Pyrrhocoris apterus, to Harvard University for experimental studies on the developing larvae. But the larvae did not develop normally as observed for many years in Prague. They changed to large size forms during the last metamorphosis and not to the insect. The containers in which the larvae were kept during development were laid out with a sheet of newspaper as was done previously in Prague. The investigators thereafter used newspaper of different origins, including Science, Nature, The Times and The Wall Street Journal. They found normal development of the larvae on European and Japanese papers and an inhibition of growth on American papers. In this way an insect-growth-inhibiting substance which occurs only in American pinewood was found.

These are some examples which I have selected. I am sure that others would be 


\section{F. Lembeck}

able to add some more, some better or some personal examples of this kind. I would say that we should keep this in mind, even in a period where technical planning in pharmacological and therapeutic research is an inevitable necessity and the only sound way to approach research. In spite of elaborate plans, many unsuccessful errors are included in the work of us all.

Several of the above mentioned examples can certainly not be regarded simply as 'errors'. They are unexpected events or lucky accidents. Such things fall on the right soil only when they come into the hands of experienced research workers who are trained to work on a profound experimental basis, being experts in their fields and possessing the full ability to evaluate an unexpected new result. All those research workers I mentioned in my examples were indeed outstanding scientists.

Jacob von Uexküll once referred in a discussion to the pessimistic epigram that the truth of today is possibly no more than the error of tomorrow. Otto Koehler, however, replied: 'No,-the truth of today is of no significance before tomorrow!' I would like to add: 'An error of today can, although only very rarely, turn out to be an unexpected success tomorrow'. We cannot search for it, but we must be aware of its possible occurrence.

I conclude my remarks by citing a distinguished pharmacologist who mentioned some of these examples some years ago, and by referring to a well-known poet.

Sir John Gaddum wrote in his very clear manner: 'It will probably always be more important to try a thing out than to argue about it.'

Erich Kästner gave us a comment in a much broader sense when he wrote:

Errors are quite good, But only here and there, Not everyone

Who sets out for India

Successfully discovers America. 